\title{
Talking About My Generation: The Influence of Age on Counterfeit Luxury Consumption in the GCC Countries: An Abstract
}

\author{
Julia Pueschel, Béatrice Parguel, Cécile Chamaret, \\ and Pierre Valette-Florence
}

\begin{abstract}
The consensus in research suggests a negative correlation between consumer age and counterfeit consumption, explained by younger consumers' lower income and different values. This study explores this culture-based explanation further by considering the influence of age on counterfeit consumption in the GCC countries, controlling for differences in income. A pilot study reveals an unexpected positive correlation between age and counterfeit consumption. Drawing on the functional theories of attitudes (Katz 1960), a qualitative study of 25 UAE (United Arab Emirates) female consumers examines the psychological functions served by UAE consumers' attitudes toward genuine and counterfeit luxury goods. The findings show important generational differences in the psychological functions served by attitudes toward genuine and counterfeit luxury goods. A clear distinction emerges between attitudes of the pre-oil generation that serve more utilitarian and ego-defensive functions, while the post-oil generation's attitudes serve more hedonic and social-adjustive functions. Results suggest that public policy makers and luxury brand managers fighting counterfeiting in the region should tailor their segmentation, communication, and overall strategy according to the generation targeted. This study expands the research on counterfeit luxury goods and contributes to more detailed knowledge of the functional theories of attitudes enhancing the existing research in international management and marketing.
\end{abstract}

\author{
J. Pueschel $(\triangle) \cdot$ B. Parguel \\ Paris-Dauphine University, Paris, France \\ e-mail: beatrice.parguel@dauphine.fr \\ C. Chamaret \\ École Polytechnique, Université Paris-Saclay, Paris, France \\ e-mail: cecile.chamaret@gmail.com \\ P. Valette-Florence \\ Pierre Mendès France University, Grenoble, France \\ e-mail: pvalette@upmf-grenoble.fr
}

